\title{
BIOACTIVE POLYPHENOLIC COMPOUNDS FROM MOTHERWORT AND HAWTHORN HYDROETHANOLIC EXTRACTS
}

\author{
ANDREEA-MIRUNA NEAGUa, CORNELIU-MIRCEA CODREANU ${ }^{b}$, \\ VASILE STAICU ${ }^{\mathrm{a}, \mathrm{c}}$, RALUCA STAN ${ }^{\mathrm{a},{ }^{*}}$
}

\begin{abstract}
Motherwort (Leonurus cardiaca) and Hawthorn (Crataegus monogyna) are known from traditional phytotherapy as being effective in alleviating many cardiovascular system disorders. This paper presents the studies made to assess the phytochemical compozition of the herbal extracts prepared from Motherwort (aerial parts), Hawthorn (fruits) and Hawthorn (flowers and leaves) by maceration, percolation and reflux using a mixture of ethanol and water as solvent. Optimum extraction conditions were evaluated for enhancing the content of the active substances (certain flavones and polyphenols: anthocyanins, etc.) present in the two studied plants, that are useful in the prevention of many cardiovascular disorders. Their chemical composition was investigated using UV-VIS spectroscopy methods: total polyphenolic content, total flavones content and the antioxidant activity were determined. The study showed that reflux extraction technique is more effective for extracting polyphenolic compounds from the two studied medicinal plants. Varying the extraction parameters led to the attainment of new phytotherapeutic extracts that are optimally beneficial in the phytotherapy of cardiovascular diseases.
\end{abstract}

Keywords: Hawthorn, Motherwort, reflux, percolation, maceration, polyphenols

\section{INTRODUCTION}

Hawthorn (Crataegus monogyna) belongs to the Rosaceae family and it is found in Europe, East Asia and eastern North America. Hawthorn fruits, leaves and flowers have been used as food and medicine around the world for centuries, being the most potent parts of the plant. Their chemical

a University Politehnica of Bucharest, Faculty of Applied Chemistry and Materials Science, 1-7 Gheorghe Polizu Street, district 1, RO-011061, Bucharest, Romania

b "Dunarea de Jos" University of Galati, Faculty of Physical Education and Sport, 63-65 Garii Street, RO-800003, Galati, Romania

${ }^{c}$ Research and Development Department, S.C. Hofigal Export-Import S.A., 2 Intrarea Serelor Street, district 4, RO-042124, Bucharest, Romania

*Corresponding author: rl_stan2000@yahoo.com 
composition includes flavonoids, triterpene saponins and amines which confer cardiotonic, diuretic and antiatherosclerotic properties. Past and ongoing studies also suggest that flavonoids, particularly oligomeric proanthocyanidins present in the hawthorn extract, are the major bioactive constituents that possess potent antioxidant activity. Hawthorn extract has the potential to be a viable, natural alternative treatment for heart disease, as well as a promoter of general cardiovascular health [1,2].

Motherwort (Leonurus cardiaca) is a representative of the Lamiaceae family and it is native to Asia and southeastern Europe. It has been proven to be very effective in treating several cardiovascular disorders and some femalespecific diseases due to its composition (sterols, alkaloids, furanic diterpenes and iridoids) that provides a complex biological activity (antimicrobial, analgesic, antioxidant, cardioprotective) [3,4].

Cardiovascular diseases are a group of disorders of the heart and blood vessels that, according to the World Health Organization, are the main source of death around the world. In 2016, around 17.9 million people died from cardiovascular diseases. Albeit these conditions stay pervasive in worldwide death rates, people can begin finding a way to forestall them [5]. The potential application in alleviating several cardiac disorders, as well as female-specific afflictions, made Motherwort and Hawthorn very good candidates for the development of alternative treatments.

The bioactive potential of Motherwort and Hawthorn are for the most part related to their phenolic constituents, which are usually the main components in the polar extracts. The antioxidant capacity of extracts acquired with polar solvents from these two plants has been recently evaluated through chemical antiradical assays [6].

The present paper aims to investigate the relationship between the antioxidant properties and the specific phenolic composition of some natural extracts from Hawthorn (fruits), Hawthorn (flowers and leaves) and Motherwort (aerial parts). Hawthorn flowers were extracted together with the leaves. That is due to the fact that Hawthorn leaf and flower have been subject to numerous clinical trials demonstrating the usefulness of Hawthorn leaf and flower extract in the management of cardiovascular diseases [7].The individual herbal extracts are known from traditional phytotherapy as being effective in alleviating many cardiovascular system disorders.

\section{RESULTS AND DISCUSSION}

Polyphenolic phytochemicals are of great interest due to the recognition of their antioxidant capacities and their great abundance in our diet and in medicinal plants. Polyphenols include flavonoids, phenolic acids, stilbenes and lignans. 
Flavonoids are naturally occurring antioxidants widely present in fruits, vegetables and beverages (tea, wine, juice, etc.). They may be divided into several subclasses such as flavanols (catechin and proanthocyanidin), flavanones (naringin and hesperidin), isoflavanones, flavonols (quercetin and myricetin), flavones (luteolin and apigenin) and anthocyanidins. Epidemiologic studies have shown an association between the intake of flavonoids and reduced risks of cardiovascular diseases, such as myocardial infarction and stroke. It appears to be attributed to their antioxidant properties [8].

Anthocyanins are water-soluble secondary plant metabolites that are responsible for making bright-colored flowers and fruits attractive to pollinators or animals. Anthocyanins also protect cells from damage due to exposure to UV-light. They may also act as antioxidants in the cell vacuoles. Plants rich in anthocyanins include blueberries, cranberries, raspberries, blackberries, strawberries, cherries and grapes, among many other species.

The correlation between the total polyphenolic constituents of Motherwort and Hawthorn and the bioactive properties of their hydroethanolic extracts was herein evaluated.

In the present study, the phytochemical profile of hydroethanolic extracts of Motherwort (aerial parts), Hawthorn (fruits) and Hawthorn (flowers and leaves) was analyzed using UV-Vis Spectrophotometry. The results of the qualitative and quantitative analyses are summarized in Figures 1-10. Data are reported as the average of three analyses.

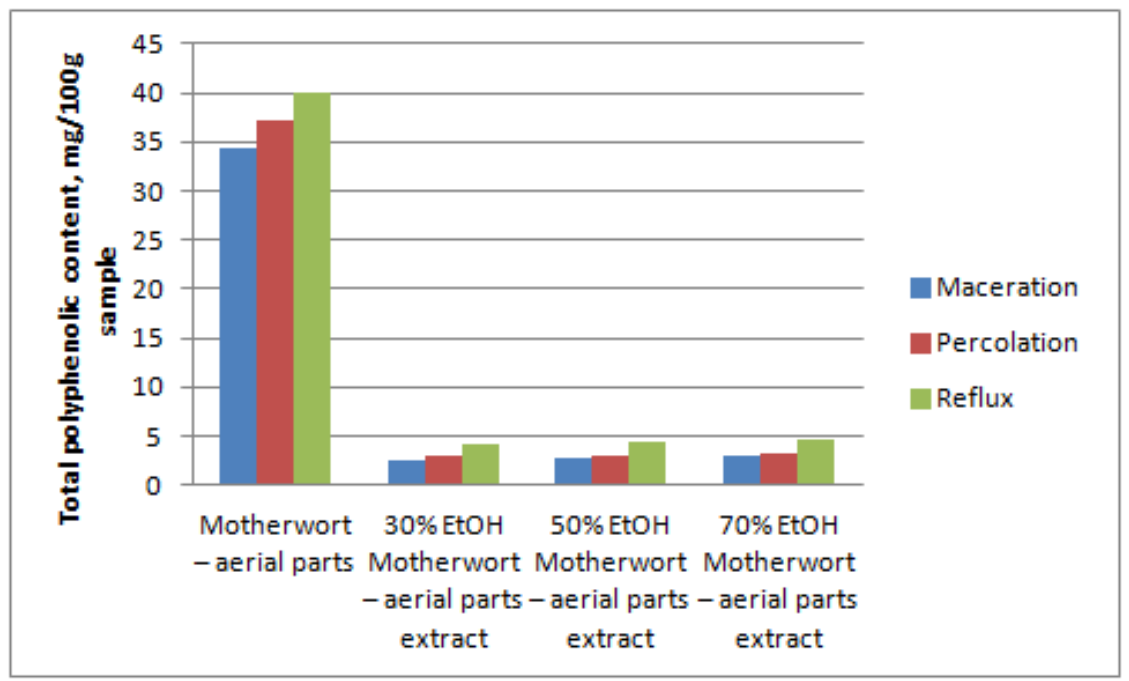

Figure 1. Total polyphenolic content from Motherwort - aerial parts and Motherwort - aerial parts extracts, expressed as gallic acid 


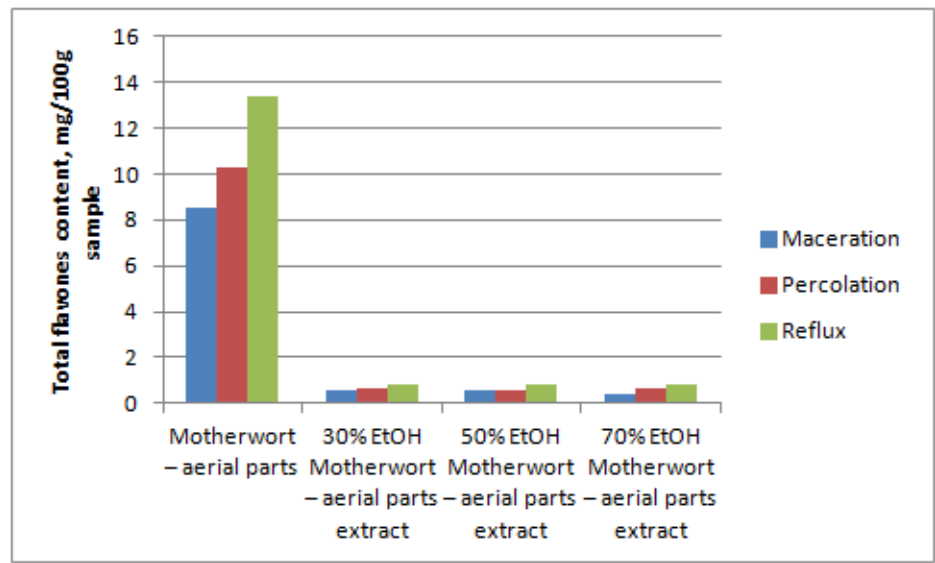

Figure 2. Total flavones content from Motherwort - aerial parts and Motherwort - aerial parts extracts, expressed as rutin

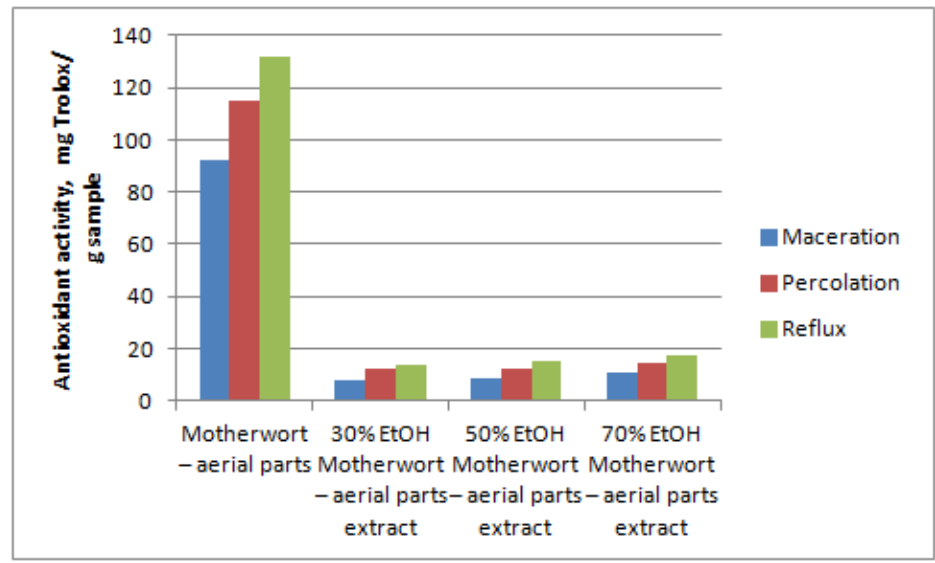

Figure 3. Antioxidant activity of Motherwort - aerial parts and Motherwort - aerial parts extracts, expressed as Trolox equiv.

Figures 1-3 depict the results for the Motherwort - aerial parts and Motherwort - aerial parts extracts for polyphenols, flavones and the antioxidant activity. According to reported studies, the total polyphenolic content in Motherwort - aerial parts extracts is between 0.4 and $7.3 \mathrm{mg} / 100 \mathrm{~g}$ sample, so the values obtained in our study are above this range [3]. The best results were obtained for the $70 \%$ ethanol extracts (total polyphenolic content - approx. $5 \mathrm{mg} / 100 \mathrm{~g}$ sample, total flavonoid content - approx. $1 \mathrm{mg} / 100 \mathrm{~g}$ sample). Reflux with $70 \%$ ethanol is the most suitable extraction method of the Motherwort aerial parts. It promoted the highest contents of total phenolics, total flavonoids, major active compounds, and the most potent antioxidant activity. 


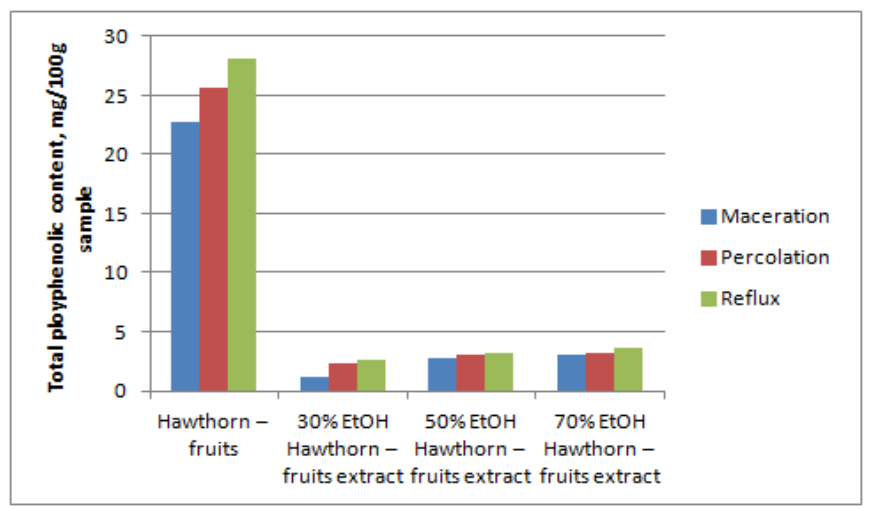

Figure 4. Total polyphenolic content from Hawthorn - fruits and Hawthorn fruits extracts, expressed as gallic acid

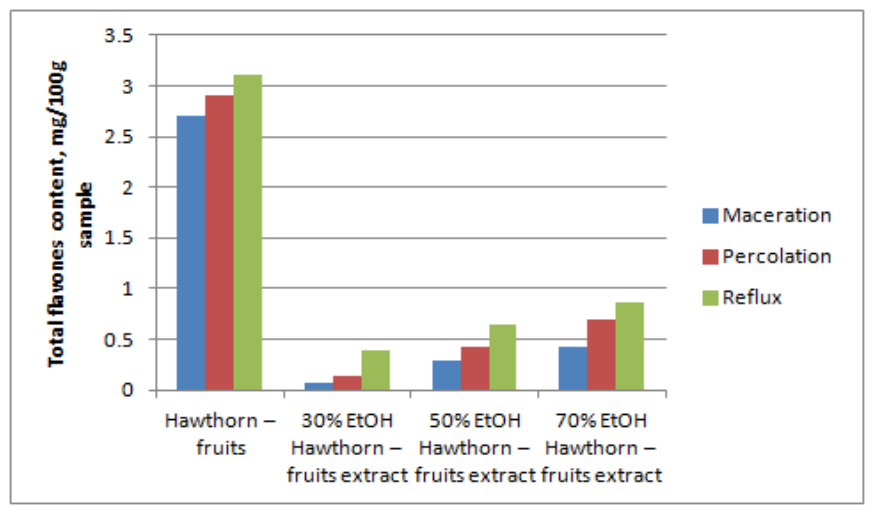

Figure 5. Total flavones content from Hawthorn - fruits and Hawthorn fruits extracts, expressed as rutin

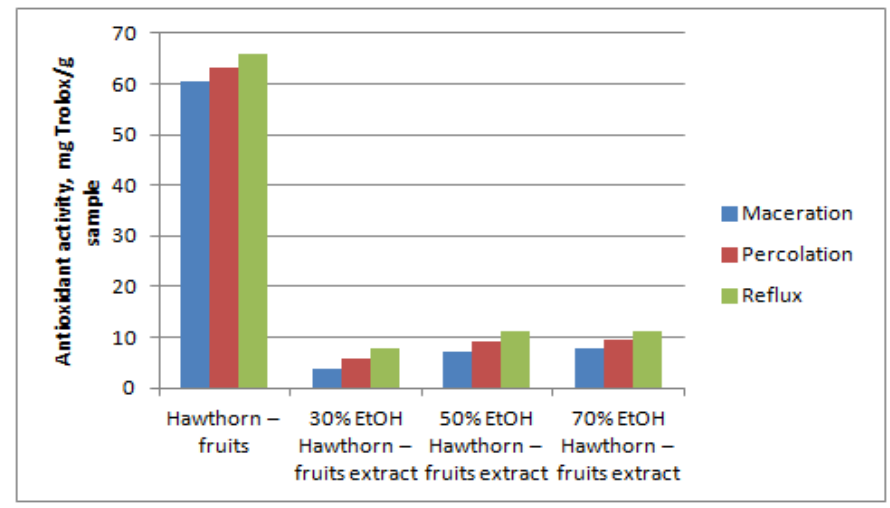

Figure 6. Antioxidant activity of Hawthorn - fruits and Hawthorn fruits extracts, expressed as Trolox equiv. 


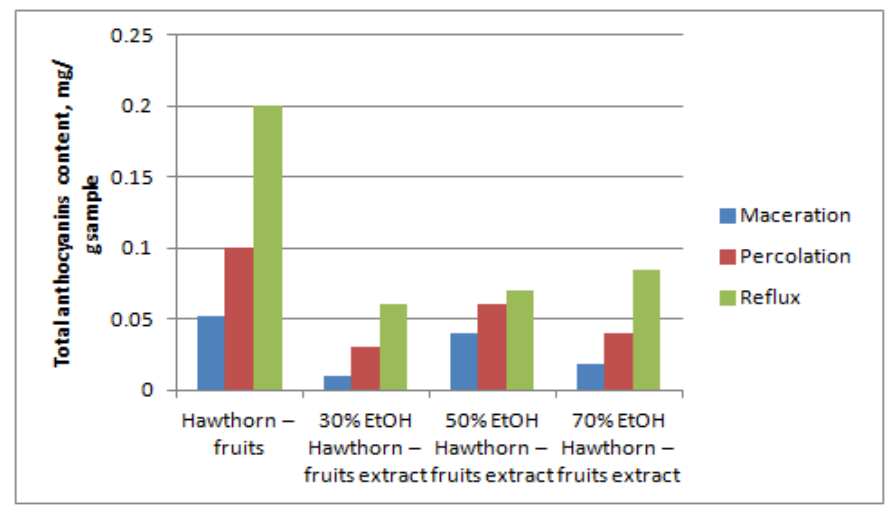

Figure 7. Total anthocyanins content from Hawthorn - fruits and Hawthorn fruits extracts, expressed as cyanidin 3-O-glucoside chloride

Figures 4-7 depict the results for the Hawthorn - fruits and Hawthorn - fruits extracts for polyphenols, flavones, anthocyanins and the antioxidant activity. Polyphenols have similar values in the $50 \%$ and $70 \%$ ethanol extracts, but much lower values in the $30 \%$ ethanol extracts, also the results are higher for the reflux extracts. In addition to total polyphenolic content, spectrophotometric methods are also used in the determination of total contents of individual groups of phenolic compounds, such as total flavones and total content of anthocyanins as cyanidin 3-glucoside. Flavones determination show better results for the reflux-obtained extracts, ranging from 0.4 to $0.8 \mathrm{mg} / 100 \mathrm{~g}$ sample. As for the total content of anthocyanins in the Hawthorn - fruits extracts, we can note better extraction by percolation in $50 \%$ ethanol solution $(0.06 \mathrm{mg} / \mathrm{g}$ sample) and by reflux in $70 \%$ ethanol solution (0.09 $\mathrm{mg} / \mathrm{g}$ sample).

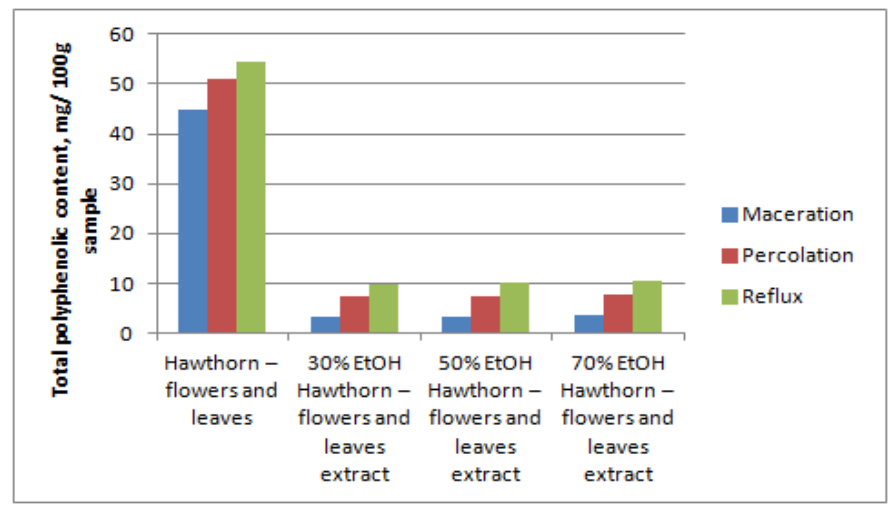

Figure 8. Total polyphenolic content from Hawthorn - flowers and leaves and Hawthorn - flowers and leaves extracts, expressed as gallic acid 


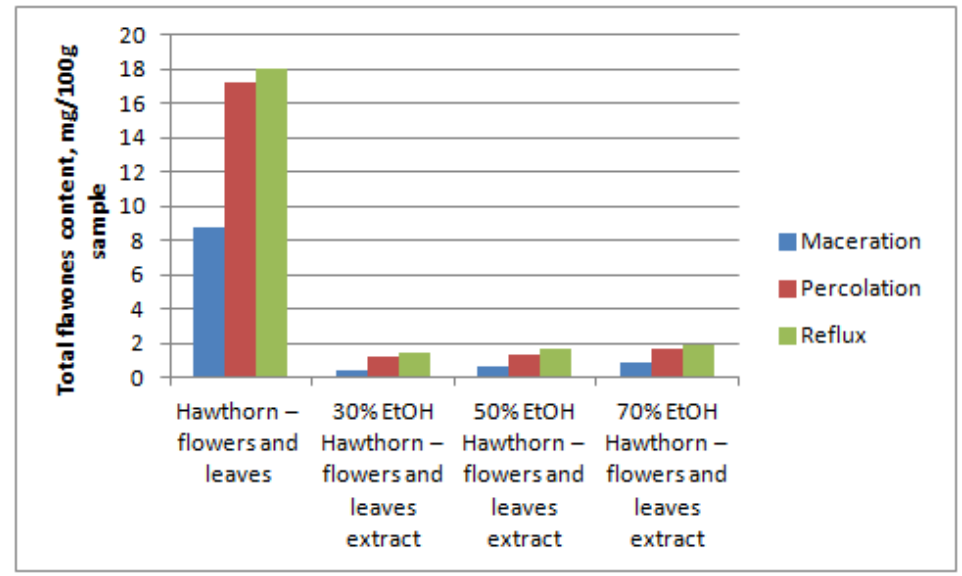

Figure 9. Total flavones content from Hawthorn - flowers and leaves and Hawthorn - flowers and leaves extracts, expressed as rutin

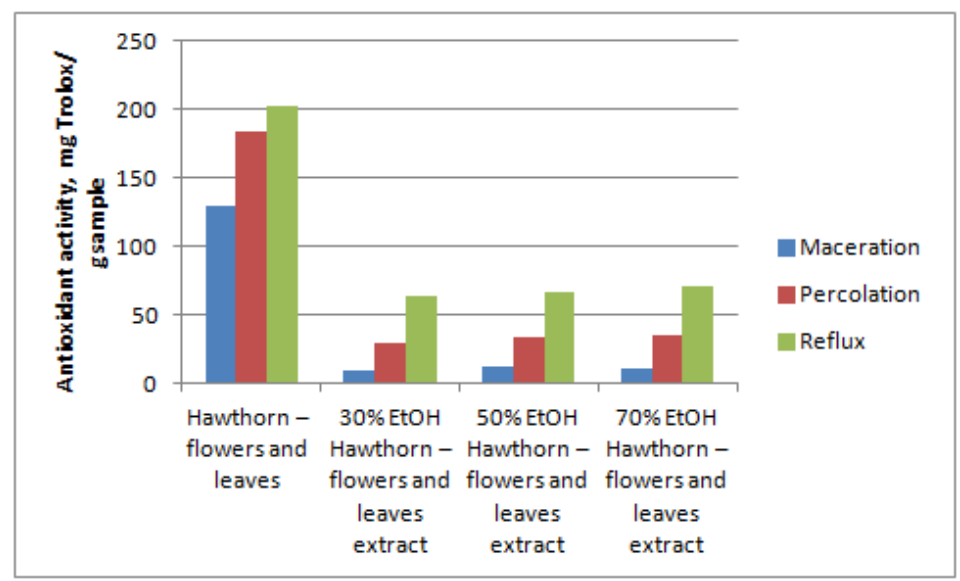

Figure 10. Antioxidant activity of Hawthorn - flowers and leaves and Hawthorn flowers and leaves extracts, expressed as Trolox equiv.

Figures 8-10 depict the results for the Hawthorn - flowers and leaves and Hawthorn - flowers and leaves extracts for polyphenols, flavones and the antioxidant activity. They have similar values regardless the extraction method applyed, with higher values for the total polyphenolic content of about $10 \mathrm{mg} / 100 \mathrm{~g}$ sample.

The hydroethanolic extracts of Hawthorn (fruits), Hawthorn (flowers and leaves) and Motherwort (aerial parts) exhibited promising antioxidant potential, as evaluated by the CUPRAC assay, with potencies order of Hawthorn (flowers and leaves) > Motherwort (aerial parts) > Hawthorn 
(fruits) and values ranging from 3.6 to $70.5 \mathrm{mg}$ Trolox/g sample. The results for the antioxidant activity of the studied extracts indicate the influence of the polyphenols and flavones present in each extract.

All in all, it can clearly be stated that the pharmacological activities of the examined traditionally used medicinal plants cannot be traced to one active compound but have to be regarded as the orchestrated effect of the entire spectrum of their phenolic constituents.

\section{CONCLUSIONS}

A detailed quantitative analysis of the phenolic compound spectrum of both Motherwort (Leonurus cardiaca) and Hawthorn (Crataegus monogyna) was conducted in order to understand their pharmacological activity.

The results show an increased content of anthocyanins in ethyl alcohol:water solution 50:50 VN Hawthorn - fructus extract. Also, we can observe that reflux extraction technique is more effective for extracting polyphenols and flavones from the two studied medicinal plants. Focusing on the phenolic characteristics of the most-active extracts, their content in flavones and in anthocyanins is possibly connected with the antioxidant capacity of Motherwort and Hawthorn, respectively. Therefore, the extracts are considered as helpful sources of natural metabolites with possible health-benefit properties.

Varying the extraction parameters led to the attainment of new phytotherapeutic extracts that are optimally beneficial in the phytotherapy of cardiovascular diseases.

\section{EXPERIMENTAL SECTION}

UV-VIS Spectrophotometry was performed on a Jasco V-530 UVVisible Spectrophotometer, with $2.0 \mathrm{~nm}$ Resolution and Double-beam Configuration.

\section{Extraction of phenolic compounds:}

The extracts were prepared from Motherwort (aerial parts), Hawthorn (fruits) and Hawthorn (flowers and leaves). The Hawthorn flowers and leaves were used as a mixture for the extraction in a 1:1.5 flowers:leaves ratio.

The plant samples of Motherwort (aerial parts), Hawthorn (fruits) and Hawthorn (flowers and leaves) were extracted in order to determine the total flavones content, total polyphenolic content and the antioxidant activity using the following method: an extract was prepared in a 1:10 of plant:solvent ratio in $50 \%$ ethanol by refluxing for 30 minutes. The obtained extract was filtered hot. 
In order to determine total anthocyanins content from Hawthorn (fruits), the sample was previously submitted to extraction with methanol solvent as it follows: an extract was prepared in a 0.5:9.5 of plant:solvent ratio in methanol by mechanically stirring for 30 minutes. The obtained extract was filtered and diluted to $100 \mathrm{~mL}$ with methanol in volumetric flask. After that, a 50 -fold dilution of this solution was prepared in a $0.1 \% \mathrm{~V} / \mathrm{V}$ solution of hydrochloric acid in methanol.

The herbal extracts from Motherwort (aerial parts), Hawthorn (fruits) and Hawthorn (flowers and leaves) were obtained by conventional extraction methods as shown in Table 1. The plant:solvent ratio used depends on the actual volume of the dried, powdered plant material. Therefore, based on our previous experiments, we used the volume of solvent that completely soaked the powdered plant material and added excess to completely immerse.

Table 1. Experimental conditions for the three conventional methods used

\begin{tabular}{|l|c|c|c|}
\hline \multicolumn{4}{|c|}{ Extraction technique } \\
\hline Solvent used & Maceration & Percolation & Reflux \\
\hline Plant:solvent ratio & $\begin{array}{c}30 \% / 50 \% / 70 \% \\
\text { ethanol solution }\end{array}$ & $\begin{array}{c}30 \% / 50 \% / 70 \% \\
\text { ethanol solution }\end{array}$ & $\begin{array}{c}30 \% / 50 \% / 70 \% \\
\text { ethanol solution }\end{array}$ \\
\hline Temperature & $1: 7$ & $1: 15$ & $1: 10$ \\
\hline Pressure applied & Not applicable & Not applicable & Not applicable \\
\hline Time required & 6 days & 6 days & 30 minutes \\
\hline $\begin{array}{l}\text { Volume of solvent } \\
\text { required }\end{array}$ & $500 \mathrm{~mL}$ & $500 \mathrm{~mL}$ & $50 \mathrm{~mL}$ \\
\hline
\end{tabular}

Determination of total polyphenolic content expressed as gallic acid equivalents: the polyphenols in the extracts are determined colorimetrically using Folin-Ciocalteu phenol reagent [9].

Determination of total flavones content expressed as rutin equivalents: the flavone derivatives content was determined by the reaction with aluminum chloride and total content was expressed in rutin [10].

Determination of total content of anthocyanins as cyanidin 3glucoside: in the European Pharmacopoeia, a specification for total content of anthocyanins, calculated as cyanidin 3-glucoside, is given in the monographs for fresh bilberry fruit and fresh bilberry fruit dry extract, refined and standardized. The latter monograph also specifies a maximum limit for anthocyanidins, calculated as cyanidin, describes a certain chromatographic profile of 15 anthocyanins and 5 anthocyanidins to confirm identity [11]. 
Determination of antioxidant activity: the CUPRAC - cupric ion reducing antioxidant capacity - method is a simple and versatile antioxidant capacity assay useful for a wide variety of polyphenols, including phenolic acids, hydroxycinnamic acids, flavonoids, carotenoids, anthocyanins, as well as for thiols, synthetic antioxidants, and vitamins $C$ and $E$. This method is based on electron transfer - it detects the ability of a potential antioxidant substance to transfer an electron to reduce any compound (Figure 11). The calibration curve was performed with a Trolox solution of known concentrations, $10-60 \mu \mathrm{g} / \mathrm{mL}[12]$.

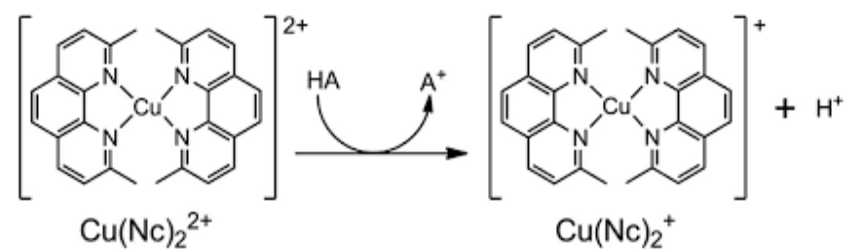

Figure 11. Reaction mechanism of copper reducing antioxidant capacity assay (CUPRAC)

\section{REFERENCES}

1. W-T. Chang; J. Dao; Z-H. Shao; Am. J, Chin. Med., 2005, 33, 1-10.

2. L. Barros; A. M. Carvalho; I. C. F. R. Ferreira; Phytochem. Anal., 2011, 22, 181-188.

3. R. C. Fierascu; I. Fierascu; A. Ortan; I. C. Fierascu; V. Anuta; B. S. Velescu; S. M. Pituru; C. E. Dinu-Pirvu; BioMed Res. Int., 2019, 2, 1-13.

4. B. Sadowska; B. Micota; M. Rozalski; M. Redzynia; M. Rozalski; Innate Immun., 2017, 0, 1-10.

5. J. Stewart; G. Manmathan; P. Wilkinson; JR. Soc. Med. Cardiovasc. Dis., 2017, 0, 1-9.

6. A. Altemimi; N. Lakhssassi; A. Baharlouei; D. G. Watson; D. A. Lightfoot; Plants, 2017, 42, 1-23.

7. Hawthorn leaf and flower, Crataegus spp., folium cum flore; European Medicines Agency, 2016, 268358.

8. M. L. McCullough; J. J. Peterson; R. Patel; P. F. Jacques; R. Shah; J. T. Dwyer; Am. J. Clin. Nutr., 2012, 95, 454-464.

9. ISO 14502-1:2005(E), Determination of substances characteristic of green and black tea - Part 1: Content of total polyphenols in tea - Colorimetric method using Folin-Ciocalteu reagent.

10. L. Silva; B. Pezzini; L. Soares; Pharmacogn. Mag., 2015, 11, 96-101.

11. Bilberry Fruit, fresh, Monograph, in European Pharmacopoeia, 2017, I, 1173.

12. R. Apak; K. Güçlü; M. Özyürek; S. Esi n Karademi r; M. Altun; Free Radic. Res., 2005, 39, 949-961. 